\title{
On Infinitary Rational Relations and Borel Sets
}

\author{
Olivier Finkel \\ Equipe de Logique Mathématique \\ U.F.R. de Mathématiques, Université Paris 7 \\ 2 Place Jussieu 75251 Paris cedex 05, France \\ finkel@logique.jussieu.fr.
}

\begin{abstract}
We prove in this paper that there exists some infinitary rational relations which are $\boldsymbol{\Sigma}_{\mathbf{3}}^{\mathbf{0}}$-complete Borel sets and some others which are $\boldsymbol{\Pi}_{3}^{\mathbf{0}}$-complete. These results give additional answers to questions of Simonnet Sim92] and of Lescow and Thomas [Tho90] LT94].
\end{abstract}

Keywords: infinitary rational relations; topological properties; Borel sets.

\section{Introduction}

Acceptance of infinite words by finite automata was firstly considered in the sixties by Büchi in order to study decidability of the monadic second order theory of one successor over the integers Büc62. Then the so called $\omega$-regular languages have been intensively studied and many applications have been found, see [Tho90] Sta97] [PP01] for many results and references.

Since then many extensions of $\omega$-regular languages have been investigated as the classes of $\omega$-languages accepted by pushdown automata, Petri nets, Turing machines, see Tho90] EH93] Sta97] for a survey of this work.

On the other side rational relations on finite words were studied in the sixties and played a fundamental role in the study of families of context free languages Ber79. Investigations on their extension to rational relations on infinite words were carried out or mentioned in the books [BT70 [LS77. Gire and Nivat studied infinitary rational relations in Gir81 GN84. Infinitary rational relations are subsets of $\Sigma_{1}^{\omega} \times \Sigma_{2}^{\omega}$, where $\Sigma_{1}$ and $\Sigma_{2}$ are finite alphabets, which are recognized by Büchi transducers or by 2-tape finite Büchi automata with asynchronous reading heads. So the class of infinitary rational relations extends both the class of finitary rational relations and the class of $\omega$-regular languages.

They have been much studied, in particular in connection with the rational functions they may define, see for example CG99 BCPS00] Sim92 Sta97 Pri00] for many results and references. Notice that a rational relation $R \subseteq \Sigma_{1}^{\omega} \times \Sigma_{2}^{\omega}$ may be seen as an $\omega$-language over the alphabet $\Sigma_{1} \times \Sigma_{2}$.

A way to study the complexity of languages of infinite words accepted by finite machines is to study their topological complexity and firstly to locate them with regard to the Borel and the projective hierarchies. This work is analysed for 
example in [Sta86] Tho90] EH93] LT94 [Sta97]. It is well known that every $\omega$-language accepted by a Turing machine with a Büchi or Muller acceptance condition is an analytic set and that $\omega$-regular languages are boolean combinations of $\boldsymbol{\Pi}_{2}^{\mathbf{0}}$-sets hence $\boldsymbol{\Delta}_{3}^{\mathbf{0}}$-sets, [Sta97] [PP01].

The question of the topological complexity of relations on infinite words also naturally arises and is asked by Simonnet in Sim92. It is also posed in a more general form by Lescow and Thomas in [LT94] (for infinite labelled partial orders) and in [Tho89] where Thomas suggested to study reducibility notions and associated completeness results.

Every infinitary rational relation is an analytic set. We showed in Fin01b that there exist some infinitary rational relations which are analytic but non Borel sets. Considering Borel infinitary rational relations we prove in this paper that there exist some infinitary rational relations which are $\boldsymbol{\Sigma}_{3}^{0}$-complete Borel sets and some others which are $\boldsymbol{\Pi}_{3}^{0}$-complete. This implies that there exist also some infinitary rational relations which are $\boldsymbol{\Delta}_{\mathbf{4}}^{\mathbf{0}}$-sets but not $\left(\boldsymbol{\Sigma}_{\mathbf{3}}^{\mathbf{0}} \cup \boldsymbol{\Pi}_{\mathbf{3}}^{\mathbf{0}}\right)$-sets.

These results may be compared with examples of $\Sigma_{3}^{0}$-complete $\omega$-languages accepted by deterministic pushdown automata with the acceptance condition: "some stack content appears infinitely often during an infinite run", given by Cachat, Duparc, and Thomas in CDT02 or with examples of $\boldsymbol{\Sigma}_{\mathbf{n}}^{\mathbf{0}}$-complete and $\Pi_{\mathbf{n}}^{\mathbf{0}}$-complete $\omega$-languages, $n \geq 1$, accepted by non-deterministic pushdown automata with Büchi acceptance condition given in [Fin01a.

The paper is organized as follows. In section 2 we introduce the notion of transducers and of infinitary rational relations. In section 3 we recall definitions of Borel sets, and we prove our main results in sections 4 and 5 .

\section{Infinitary rational relations}

Let $\Sigma$ be a finite alphabet whose elements are called letters. A non-empty finite word over $\Sigma$ is a finite sequence of letters: $x=a_{1} a_{2} \ldots a_{n}$ where for all $i$ in $[1 ; n]$, $a_{i} \in \Sigma$. We shall denote $x(i)=a_{i}$ the $i^{\text {th }}$ letter of $x$ and $x[i]=x(1) \ldots x(i)$ for $i \leq n$. The length of $x$ is $|x|=n$. The empty word will be denoted by $\lambda$ and has 0 letter. Its length is 0 . The set of finite words over $\Sigma$ is denoted $\Sigma^{\star}$. A (finitary) language $L$ over $\Sigma$ is a subset of $\Sigma^{\star}$. The usual concatenation product of $u$ and $v$ will be denoted by $u . v$ or just $u v$. For $V \subseteq \Sigma^{\star}$, we denote $V^{\star}=\left\{v_{1} \ldots v_{n} \mid n \in \mathbb{N} \quad\right.$ and $\left.\quad \forall i \in[1 ; n] \quad v_{i} \in V\right\}$.

The first infinite ordinal is $\omega$. An $\omega$-word over $\Sigma$ is an $\omega$-sequence $a_{1} a_{2} \ldots a_{n} \ldots$, where for all $i \geq 1, a_{i} \in \Sigma$. When $\sigma$ is an $\omega$-word over $\Sigma$, we write $\sigma=$ $\sigma(1) \sigma(2) \ldots \sigma(n) \ldots$ and $\sigma[n]=\sigma(1) \sigma(2) \ldots \sigma(n)$ the finite word of length $n$, prefix of $\sigma$. The set of $\omega$-words over the alphabet $\Sigma$ is denoted by $\Sigma^{\omega}$. An $\omega$-language over an alphabet $\Sigma$ is a subset of $\Sigma^{\omega}$. For $V \subseteq \Sigma^{\star}, V^{\omega}=\{\sigma=$ $\left.u_{1} \ldots u_{n} \ldots \in \Sigma^{\omega} \mid \forall i \geq 1 u_{i} \in V\right\}$ is the $\omega$-power of $V$. The concatenation product is extended to the product of a finite word $u$ and an $\omega$-word $v$ : the infinite word $u . v$ is then the $\omega$-word such that: $(u . v)(k)=u(k)$ if $k \leq|u|$, and $(u . v)(k)=v(k-|u|)$ if $k>|u|$. 
If $A$ is a subset of $B$ we shall denote $A^{-}=B-A$ the complement of $A$ (in $B$ ).

We assume the reader to be familiar with the theory of formal languages and of $\omega$-regular languages. We recall that $\omega$-regular languages form the class of $\omega$-languages accepted by finite automata with a Büchi acceptance condition and this class is the omega Kleene closure of the class of regular finitary languages.

We are going now to introduce the notion of infinitary rational relation via definition of Büchi transducers:

Definition 1. A Büchi transducer is a sextuple $\mathcal{T}=\left(K, \Sigma, \Gamma, \Delta, q_{0}, F\right)$, where $K$ is a finite set of states, $\Sigma$ and $\Gamma$ are finite sets called the input and the output alphabets, $\Delta$ is a finite subset of $K \times \Sigma^{\star} \times \Gamma^{\star} \times K$ called the set of transitions, $q_{0}$ is the initial state, and $F \subseteq K$ is the set of accepting states.

A computation $\mathcal{C}$ of the transducer $\mathcal{T}$ is an infinite sequence of transitions

$$
\left(q_{0}, u_{1}, v_{1}, q_{1}\right),\left(q_{1}, u_{2}, v_{2}, q_{2}\right), \ldots\left(q_{i-1}, u_{i}, v_{i}, q_{i}\right),\left(q_{i}, u_{i+1}, v_{i+1}, q_{i+1}\right), \ldots
$$

The computation is said to be successful iff there exists a final state $q_{f} \in F$ and infinitely many integers $i \geq 0$ such that $q_{i}=q_{f}$.

The input word of the computation is $u=u_{1} \cdot u_{2} \cdot u_{3} \ldots$

The output word of the computation is $v=v_{1} \cdot v_{2} \cdot v_{3} \ldots$

Then the input and the output words may be finite or infinite.

The infinitary rational relation $R(\mathcal{T}) \subseteq \Sigma^{\omega} \times \Gamma^{\omega}$ recognized by the Büchi transducer $\mathcal{T}$ is the set of pairs $(u, v) \in \Sigma^{\omega} \times \Gamma^{\omega}$ such that $u$ and $v$ are the input and the output words of some successful computation $\mathcal{C}$ of $\mathcal{T}$.

The set of infinitary rational relations will be denoted $R A T$.

Remark 2. An infinitary rational relation is a subset of $\Sigma^{\omega} \times \Gamma^{\omega}$ for two finite alphabets $\Sigma$ and $\Gamma$. One can also consider that it is an $\omega$-language over the finite alphabet $\Sigma \times \Gamma$. If $(u, v) \in \Sigma^{\omega} \times \Gamma^{\omega}$, one can consider this pair of infinite words as a single infinite word $(u(1), v(1)) .(u(2), v(2)) .(u(3), v(3)) \ldots$ over the alphabet $\Sigma \times \Gamma$. We shall use this fact to investigate the topological complexity of infinitary rational relations.

\section{Borel sets}

We assume the reader to be familiar with basic notions of topology which may be found in Mos80] Kec95] [LT94] Sta97] PP01].

For a finite alphabet $X$ we shall consider $X^{\omega}$ as a topological space with the Cantor topology. The open sets of $X^{\omega}$ are the sets in the form $W . X^{\omega}$, where $W \subseteq X^{\star}$. A set $L \subseteq X^{\omega}$ is a closed set iff its complement $X^{\omega}-L$ is an open set. Define now the next classes of the Hierarchy of Borel sets of finite ranks:

Definition 3. The classes $\boldsymbol{\Sigma}_{\mathbf{n}}^{\mathbf{0}}$ and $\mathbf{\Pi}_{\mathbf{n}}^{\mathbf{0}}$ of the Borel Hierarchy on the topological space $X^{\omega}$ are defined as follows: 
$\Sigma_{1}^{0}$ is the class of open sets of $X^{\omega}$.

$\Pi_{1}^{0}$ is the class of closed sets of $X^{\omega}$.

And for any integer $n \geq 1$ :

$\boldsymbol{\Sigma}_{\mathbf{n}+\mathbf{1}}^{\mathbf{0}}$ is the class of countable unions of $\boldsymbol{\Pi}_{\mathbf{n}}^{\mathbf{0}}$-subsets of $X^{\omega}$.

$\boldsymbol{\Pi}_{\mathbf{n}+\mathbf{1}}^{\mathbf{0}}$ is the class of countable intersections of $\boldsymbol{\Sigma}_{\mathbf{n}}^{\mathbf{0}}$-subsets of $X^{\omega}$.

The Borel Hierarchy is also defined for transfinite levels, but we shall not need them in the present study. There are also some subsets of $X^{\omega}$ which are not Borel. In particular the class of Borel subsets of $X^{\omega}$ is strictly included into the class $\Sigma_{1}^{1}$ of analytic sets which are obtained by projection of Borel sets, see for example [Sta97] [LT94] [PP01] Kec95] for more details.

Recall also the notion of completeness with regard to reduction by continuous functions. For an integer $n \geq 1$, a set $F \subseteq X^{\omega}$ is said to be a $\boldsymbol{\Sigma}_{\mathbf{n}}^{\mathbf{0}}$ (respectively, $\boldsymbol{\Pi}_{\mathbf{n}}^{\mathbf{0}}, \boldsymbol{\Sigma}_{\mathbf{1}}^{\mathbf{1}}$ )-complete set iff for any set $E \subseteq Y^{\omega}$ (with $Y$ a finite alphabet): $E \in \boldsymbol{\Sigma}_{\mathbf{n}}^{\mathbf{0}}$ (respectively, $E \in \mathbf{\Pi}_{\mathbf{n}}^{\mathbf{0}}, E \in \boldsymbol{\Sigma}_{\mathbf{1}}^{\mathbf{1}}$ ) iff there exists a continuous function $f: Y^{\omega} \rightarrow$ $X^{\omega}$ such that $E=f^{-1}(F)$.

A $\boldsymbol{\Sigma}_{\mathbf{n}}^{\mathbf{0}}$ (respectively, $\boldsymbol{\Pi}_{\mathbf{n}}^{\mathbf{0}}, \boldsymbol{\Sigma}_{\mathbf{1}}^{\mathbf{1}}$ )-complete set is a $\boldsymbol{\Sigma}_{\mathbf{n}}^{\mathbf{0}}$ (respectively, $\boldsymbol{\Pi}_{\mathbf{n}}^{\mathbf{0}}, \boldsymbol{\Sigma}_{\mathbf{1}}^{\mathbf{1}}$ )-set which is in some sense a set of the highest topological complexity among the $\boldsymbol{\Sigma}_{\mathbf{n}}^{\mathbf{0}}$ (respectively, $\mathbf{\Pi}_{\mathbf{n}}^{\mathbf{0}}, \boldsymbol{\Sigma}_{\mathbf{1}}^{\mathbf{1}}$ )-sets. $\boldsymbol{\Sigma}_{\mathbf{n}}^{\mathbf{0}}$ (respectively, $\boldsymbol{\Pi}_{\mathbf{n}}^{\mathbf{0}}$ )-complete sets, with $n$ an integer $\geq 1$, are thoroughly characterized in Sta86].

Example 4. Let $\Sigma=\{0,1\}$ and $\mathcal{A}=\left(0^{\star} .1\right)^{\omega} \subseteq \Sigma^{\omega}$. $\mathcal{A}$ is the set of $\omega$-words over the alphabet $\Sigma$ with infinitely many occurrences of the letter 1 . It is well known that $\mathcal{A}$ is a $\mathbf{\Pi}_{\mathbf{2}}^{\mathbf{0}}$-complete set and its complement $\mathcal{A}^{-}$is a $\boldsymbol{\Sigma}_{\mathbf{2}}^{\mathbf{0}}$-complete set: it is the set of $\omega$-words over $\{0,1\}$ having only a finite number of occurrences of letter 1 .

\section{$4 \quad \Sigma_{3}^{0}$-complete infinitary rational relations}

We can now state the following result:

Theorem 5. There exist some $\boldsymbol{\Sigma}_{\mathbf{3}}^{0}$-complete infinitary rational relations.

Proof. We shall use a well known example of $\boldsymbol{\Sigma}_{3}^{0}$-complete set which is a subset of the topological space $\Sigma^{\omega^{2}}$.

The set $\Sigma^{\omega^{2}}$ is the set of $\omega^{2}$-words over the finite alphabet $\Sigma$. It may also be viewed as the set of (infinite) $(\omega \times \omega)$-matrices whose coefficients are letters of $\Sigma$. If $x \in \Sigma^{\omega^{2}}$ we shall write $x=(x(m, n))_{m \geq 1, n \geq 1}$. The infinite word $x(m, 1) x(m, 2) \ldots x(m, n) \ldots$ will be called the $m^{t h}$ column of the $\omega^{2}$-word $x$ and the infinite word $x(1, n) x(2, n) \ldots x(m, n) \ldots$ will be called the $n^{\text {th }}$ row of the $\omega^{2}$-word $x$. Thus an element of $\Sigma^{\omega^{2}}$ is completely determined by the (infinite) set of its columns or of its rows.

The set $\Sigma^{\omega^{2}}$ is usually equipped with the product topology of the dicrete topology on $\Sigma$ (for which every subset of $\Sigma$ is an open set), see [Kec95] PP01. 
This topology may be defined by the following distance $d$. Let $x$ and $y$ be two $\omega^{2}$-words in $\Sigma^{\omega^{2}}$ such that $x \neq y$, then

$$
\begin{gathered}
d(x, y)=\frac{1}{2^{n}} \quad \text { where } \\
n=\min \{p \geq 1 \mid \exists(i, j) \quad x(i, j) \neq y(i, j) \text { and } i+j=p\}
\end{gathered}
$$

Then the topological space $\Sigma^{\omega^{2}}$ is homeomorphic to the topological space $\Sigma^{\omega}$, equipped with the Cantor topology. Borel subsets of $\Sigma^{\omega^{2}}$ are defined from open subsets in the same manner as in the case of the topological space $\Sigma^{\omega}$.

$\boldsymbol{\Sigma}_{\mathbf{n}}^{\mathbf{0}}$ (respectively $\boldsymbol{\Pi}_{\mathbf{n}}^{\mathbf{0}}$ )-complete sets are also defined in a similar way.

Recall now that the set

$$
S=\left\{x \in\{0,1\}^{\omega^{2}} / \exists m \exists^{\infty} n x(m, n)=1\right\}
$$

where $\exists^{\infty}$ means "there exist infinitely many", is a $\boldsymbol{\Sigma}_{3}^{0}$-complete subset of $\{0,1\}^{\omega^{2}}$, Kec95, p. 179]. It is the set of $\omega^{2}$-words over $\{0,1\}$ having at least one column in the $\boldsymbol{\Pi}_{2}^{0}$-complete subset $\mathcal{A}$ of $\{0,1\}^{\omega}$ given in Example 4 .

In order to use this example we shall firstly define a coding of $\omega^{2}$-words over $\Sigma$ by $\omega$-words over the alphabet $(\Sigma \cup\{A\}) \times(\Sigma \cup\{A\})$ where $A$ is a new letter not in $\Sigma$. The code of $x \in \Sigma^{\omega^{2}}$ may be written in the form $\left(\sigma_{1}, \sigma_{2}\right)$, where $\sigma_{1}$ and $\sigma_{2}$ are $\omega$-words over the alphabet $(\Sigma \cup\{A\})$. In order to describe the $\omega$-words $\sigma_{1}$ and $\sigma_{2}$ let us call, for $x \in \Sigma^{\omega^{2}}$ and $p$ an integer $\geq 2$ :

$$
T_{p+1}^{x}=\{x(p, 1), x(p-1,2), \ldots, x(2, p-1), x(1, p)\}
$$

the set of elements $x(m, n)$ with $m+n=p+1$.

The word $\sigma_{1}$ begins with $x(1,1)$ followed by a letter $A$; then the word $\sigma_{1}$ enumerates the elements of the sets $T_{p+1}^{x}$ for $p$ an even integer. More precisely for every even integer $p \geq 2$ the elements of $T_{p+1}^{x}$ are placed before those of $T_{p+3}^{x}$ and these two sets of letters are separated by an $A$. Moreover for each even integer $p \geq 2$ the elements of $T_{p+1}^{x}$ are enumerated in the following order:

$$
x(p, 1), x(p-1,2), \ldots, x(2, p-1), x(1, p)
$$

The construction of $\sigma_{2}$ is very similar but with some modifications. It enumerates the elements of the sets $T_{p+1}^{x}$ for $p$ an odd integer. More precisely the word $\sigma_{2}$ begins with an $A$ then for every odd integer $p \geq 3$ the elements of $T_{p+1}^{x}$ are placed before those of $T_{p+3}^{x}$ and these two sets of letters are separated by an $A$. Moreover for each odd integer $p \geq 3$ the elements of $T_{p+1}^{x}$ are enumerated in the following order:

$$
x(1, p), x(2, p-1), \ldots, x(p-1,2), x(p, 1)
$$

Then the $\omega$-word $\sigma_{1}$ and the $\omega$-word $\sigma_{2}$ are in the following form: 
$\sigma_{1}=x(1,1) \cdot A \cdot x(2,1) x(1,2) \cdot A \cdot x(4,1) x(3,2) x(2,3) x(1,4) \cdot A \cdot x(6,1) x(5,2) \ldots$

$\sigma_{2}=A \cdot x(1,3) x(2,2) x(3,1) \cdot A \cdot x(1,5) x(2,4) x(3,3) x(4,2) x(5,1) \cdot A \cdot x(1,7) x(2,6) \ldots$

Let then $h$ be the mapping from $\Sigma^{\omega^{2}}$ into $((\Sigma \cup\{A\}) \times(\Sigma \cup\{A\}))^{\omega}$ such that, for every $\omega^{2}$-word $x$ over the alphabet $\Sigma, h(x)$ is the code $\left(\sigma_{1}, \sigma_{2}\right)$ of the $\omega^{2}$-word as defined above. It is easy to see, from the definition of $h$ and of the order of the enumeration of letters $x(m, n)$ in the code of $x \in \Sigma^{\omega^{2}}$ (they are enumerated for increasing values of $m+n)$, that $h$ is a continuous function from $\Sigma^{\omega^{2}}$ into $((\Sigma \cup\{A\}) \times(\Sigma \cup\{A\}))^{\omega}$.

Notice that we have chosen $x(1,1)$ to be the first letter of the word $\sigma_{1}$. In fact, with slight modifications in the sequel, we could have chosen the letter $x(1,1)$ to be the first letter of the word $\sigma_{2}$.

Remark that the above coding of $\omega^{2}$-words resembles the use of the Cantor pairing function as it was used to construct the complete sets $P_{i}$ and $S_{i}$ in [SW78] (see also [Sta86] or [Sta97, section 3.4]).

We now state the following lemmas:

Lemma 6. Let $\Sigma=\{0,1\}$ and $S=\left\{x \in\{0,1\}^{\omega^{2}} / \exists m \exists^{\infty} n x(m, n)=1\right\}$. Then the set

$$
\mathcal{S}=h(S) \cup\left(h\left(\Sigma^{\omega^{2}}\right)\right)^{-}
$$

is a $\boldsymbol{\Sigma}_{\mathbf{3}}^{\mathbf{0}}$-complete subset of $((\Sigma \cup\{A\}) \times(\Sigma \cup\{A\}))^{\omega}$.

Proof. We just sketch the first part of the proof.

The topological space $\Sigma^{\omega^{2}}$ is compact and the function $h$ is continuous and injective. Using these facts we can easily show that $h(S)$ is a $\Sigma_{\mathbf{3}^{0}}^{\mathbf{0}}$-subset of $((\Sigma \cup$ $\{A\}) \times(\Sigma \cup\{A\}))^{\omega}$.

On the other side $h\left(\Sigma^{\omega^{2}}\right)$ is a closed subset of $((\Sigma \cup\{A\}) \times(\Sigma \cup\{A\}))^{\omega}$. Then its complement

$$
\left(h\left(\Sigma^{\omega^{2}}\right)\right)^{-}=((\Sigma \cup\{A\}) \times(\Sigma \cup\{A\}))^{\omega}-h\left(\Sigma^{\omega^{2}}\right)
$$

is an open (i.e. a $\left.\boldsymbol{\Sigma}_{\mathbf{1}}^{\mathbf{0}}\right)$ subset of $((\Sigma \cup\{A\}) \times(\Sigma \cup\{A\}))^{\omega}$.

Now $\mathcal{S}=h(S) \cup\left(h\left(\Sigma^{\omega^{2}}\right)\right)^{-}$is the union of a $\Sigma_{3}^{0}$-set and of a $\boldsymbol{\Sigma}_{\mathbf{1}}^{0}$-set therefore

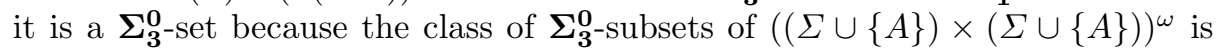
closed under finite union.

In order to prove that $\mathcal{S}$ is $\boldsymbol{\Sigma}_{3}^{0}$-complete it suffices to remark that $S=h^{-1}(\mathcal{S})$. This implies that $\mathcal{S}$ is $\boldsymbol{\Sigma}_{\mathbf{3}}^{\mathbf{0}}$-complete because $S$ is $\boldsymbol{\Sigma}_{\mathbf{3}}^{\mathbf{0}}$-complete.

Lemma 7. For $\Sigma$ a finite alphabet,

$$
\left(h\left(\Sigma^{\omega^{2}}\right)\right)^{-}=((\Sigma \cup\{A\}) \times(\Sigma \cup\{A\}))^{\omega}-h\left(\Sigma^{\omega^{2}}\right)
$$


is an infinitary rational relation.

Proof. Return to the definition of coding of $\omega^{2}$-words over $\Sigma$ by $\omega$-words over the alphabet $(\Sigma \cup\{A\}) \times(\Sigma \cup\{A\})$. The code of $x \in \Sigma^{\omega^{2}}$ was written in the form $\left(\sigma_{1}, \sigma_{2}\right)$, where $\sigma_{1}$ and $\sigma_{2}$ are $\omega$-words over the alphabet $(\Sigma \cup\{A\})$ in the form:

$\sigma_{1}=u_{1} \cdot A \cdot u_{2} \cdot A \cdot u_{4} \cdot A \cdot u_{6} \cdot A \cdot u_{8} \cdot A \ldots \cdot A \cdot u_{2 n} \cdot A \ldots$

$\sigma_{2}=A \cdot u_{3} \cdot A \cdot u_{5} \cdot A \cdot u_{7} \cdot A \cdot u_{9} \cdot A \ldots \cdot A \cdot u_{2 n+1} \cdot A \ldots$

where for all integers $i \geq 1, u_{i} \in \Sigma^{\star}$ and $\left|u_{i}\right|=i$.

It is now easy to see that the complement of the set $h\left(\Sigma^{\omega^{2}}\right)$ of codes of $\omega^{2}$-words over $\Sigma$ is the union of the sets $\mathcal{C}_{j}$ where:

$-\mathcal{C}_{1}=\left\{\left(\sigma_{1}, \sigma_{2}\right) / \sigma_{1}, \sigma_{2} \in(\Sigma \cup\{A\})^{\omega}\right.$ and $\left(\sigma_{1} \in \mathcal{B}\right.$ or $\left.\left.\sigma_{2} \in \mathcal{B}\right)\right\}$

where $\mathcal{B}$ is the set of $\omega$-words over $(\Sigma \cup\{A\})$ having only a finite number of letters $A$.

$-\mathcal{C}_{2}$ is formed by pairs $\left(\sigma_{1}, \sigma_{2}\right)$ where

$\sigma_{1}$ has not an initial segment in $\Sigma . A . \Sigma^{2} . A$ or

the first letter of $\sigma_{2}$ is not an $A$.

$-\mathcal{C}_{3}$ is formed by pairs $\left(\sigma_{1}, \sigma_{2}\right)$ where

$\sigma_{1}=w_{1} \cdot A \cdot w_{2} \cdot A \cdot w_{3} \cdot A \cdot w_{4} \ldots$ A. $w_{n} \cdot A \cdot u \cdot A \cdot z_{1}$

$\sigma_{2}=w_{1}^{\prime} \cdot A \cdot w_{2}^{\prime} \cdot A \cdot w_{3}^{\prime} \cdot A \cdot w_{4}^{\prime} \ldots$ A. $w_{n}^{\prime} \cdot A \cdot v \cdot A \cdot z_{2}$

where $n$ is an integer $\geq 1$, for all $i \leq n w_{i}, w_{i}^{\prime} \in \Sigma^{\star}, z_{1}, z_{2} \in(\Sigma \cup\{A\})^{\omega}$ and

$$
u, v \in \Sigma^{\star} \text { and }|v| \neq|u|+1
$$

- $\mathcal{C}_{4}$ is formed by pairs $\left(\sigma_{1}, \sigma_{2}\right)$ where

$\sigma_{1}=w_{1} \cdot A \cdot w_{2} \cdot A \cdot w_{3} \cdot A \cdot w_{4} \ldots \cdot A \cdot w_{n} \cdot A \cdot w_{n+1} \cdot A \cdot v \cdot A \cdot z_{1}$

$\sigma_{2}=w_{1}^{\prime} \cdot A \cdot w_{2}^{\prime} \cdot A \cdot w_{3}^{\prime} \cdot A \cdot w_{4}^{\prime} \ldots$.A. $w_{n}^{\prime} \cdot A \cdot u \cdot A \cdot z_{2}$

where $n$ is an integer $\geq 1$, for all $i \leq n w_{i}, w_{i}^{\prime} \in \Sigma^{\star}, w_{n+1} \in \Sigma^{\star}, z_{1}, z_{2} \in$ $(\Sigma \cup\{A\})^{\omega}$ and

$$
u, v \in \Sigma^{\star} \text { and }|v| \neq|u|+1
$$

Each set $\mathcal{C}_{j}, 1 \leq j \leq 4$, is easily seen to be an infinitary rational relation $\subseteq(\Sigma \cup\{A\})^{\omega} \times(\Sigma \cup\{A\})^{\omega}$ (the detailed proof is left to the reader).

The class of infinitary rational relations is closed under finite union; this follows from the fact that they are recognized by non deterministic Büchi transducers. Then

$$
\left(h\left(\Sigma^{\omega^{2}}\right)\right)^{-}=\bigcup_{1 \leq j \leq 4} \mathcal{C}_{j}
$$

is an infinitary rational relation. 
We cannot show directly that $h(S) \in R A T$ so we are now looking for a rational relation $R \subseteq((\Sigma \cup\{A\}) \times(\Sigma \cup\{A\}))^{\omega}$ (with $\left.\Sigma=\{0,1\}\right)$ such that for every $\omega^{2}$-word $x \in \Sigma^{\omega^{2}}, h(x) \in R$ if and only if $x \in S$. Then we shall have $S=h^{-1}(R)$.

We shall first describe the relation $R$ which is an $\omega$-language over the alphabet $((\Sigma \cup\{A\}) \times(\Sigma \cup\{A\}))$. Every word of $R$ may be seen as a pair $y=\left(y_{1}, y_{2}\right)$ of $\omega$-words over the alphabet $\Sigma \cup\{A\}$ and then $y$ is in $R$ if and only if it is in the form

$y_{1}=U_{k} \cdot u \cdot t(1) \cdot v_{1} \cdot A \cdot g_{1} \cdot t(3) \cdot v_{2} \cdot A \cdot g_{2} \cdot t(5) \ldots A \cdot g_{n} \cdot t(2 n+1) \cdot v_{n+1} \cdot A \ldots$

$y_{2}=V_{k} \cdot u_{1} \cdot t(2) \cdot z_{1} \cdot A \cdot u_{2} \cdot t(4) \cdot z_{2} \cdot A \ldots$. A. $u_{n} \cdot t(2 n) \cdot z_{n} \cdot A \ldots$

where $k$ is an integer $\geq 1, U_{k}, V_{k} \in\left(\Sigma^{\star} . A\right)^{k}, u=\lambda$ or $u \in \Sigma$, and for all integers $i \geq 1, t(i) \in \Sigma$ and $u_{i}, v_{i}, g_{i}, z_{i} \in \Sigma^{\star}$ and

$$
\left|v_{i}\right|=\left|u_{i}\right| \quad \text { and } \quad\left|g_{i}\right|=\left|z_{i}\right|+1
$$

and the $\omega$-word $t=t(1) t(2) \ldots t(n) \ldots$ is in the $\omega$-regular language $\mathcal{A}$ given in Example 4.

Lemma 8. The above defined relation $R$ satisfies $S=h^{-1}(R)$, i.e.:

$$
\forall x \in \Sigma^{\omega^{2}} \quad h(x) \in R \longleftrightarrow x \in S
$$

Proof. Assume first that such an $y=\left(y_{1}, y_{2}\right) \in R$ is the code $h(x)$ of an $\omega^{2}$-word $x \in \Sigma^{\omega^{2}}$. Then

$$
u \cdot t(1) \cdot v_{1}=x(2 k, 1) \cdot x(2 k-1,2) \ldots x(1,2 k)
$$

so if $u=\lambda$ then $x(2 k, 1)=t(1)$ and $\left|v_{1}\right|=2 k-1$.

And if $u \in \Sigma$ then $x(2 k-1,2)=t(1)$ and $\left|v_{1}\right|=2 k-2$.

Next

$$
u_{1} \cdot t(2) \cdot z_{1}=x(1,2 k+1) \cdot x(2,2 k) \ldots x(2 k+1,1)
$$

so if $u=\lambda$ then $\left|u_{1}\right|=\left|v_{1}\right|=2 k-1$ thus $x(2 k, 2)=t(2)$ and $\left|z_{1}\right|=1$. And if $u \in \Sigma$ then $\left|u_{1}\right|=\left|v_{1}\right|=2 k-2$ thus $x(2 k-1,3)=t(2)$ and $\left|z_{1}\right|=2$.

Moreover

$$
g_{1} \cdot t(3) \cdot v_{2}=x(2 k+2,1) \cdot x(2 k+1,2) \ldots x(1,2 k+2)
$$

so if $u=\lambda$ then $\left|g_{1}\right|=\left|z_{1}\right|+1=2$ and $t(3)=x(2 k, 3)$.

And if $u \in \Sigma$ then $\left|g_{1}\right|=\left|z_{1}\right|+1=3$ and $t(3)=x(2 k-1,4)$.

In a similar manner one can show by induction on integers $i$ that if $u=\lambda$ letters $t(i)$ are successive letters of the $(2 k)^{t h}$ column of $x$ and if $u \in \Sigma$ letters $t(i)$ are successive letters of the $(2 k-1)^{t h}$ column of $x$. 
So assume that for some integer $i \geq 1$ :

if $u=\lambda$ then $t(2 i+1)=x(2 k, 2 i+1)$, and

if $u \in \Sigma$ then $t(2 i+1)=x(2 k-1,2 i+2)$.

We know that

$$
g_{i} \cdot t(2 i+1) \cdot v_{i+1}=x(2 k+2 i, 1) \cdot x(2 k+2 i-1,2) \ldots x(1,2 k+2 i)
$$

thus $u=\lambda$ implies that $\left|v_{i+1}\right|=2 k-1$ and $u \in \Sigma$ implies that $\left|v_{i+1}\right|=2 k-2$. But it holds also that

$$
u_{i+1} \cdot t(2 i+2) \cdot z_{i+1}=x(1,2 k+2 i+1) \cdot x(2,2 k+2 i) \ldots x(2 k+2 i+1,1)
$$

So if $u=\lambda$ then $\left|u_{i+1}\right|=\left|v_{i+1}\right|=2 k-1$ and $t(2 i+2)=x(2 k, 2 i+2)$ and $\left|z_{i+1}\right|=2 i+1$.

Anf if $u \in \Sigma$ then $\left|u_{i+1}\right|=\left|v_{i+1}\right|=2 k-2$ and $t(2 i+2)=x(2 k-1,2 i+3)$ and $\left|z_{i+1}\right|=2 i+2$.

Next

$$
g_{i+1} \cdot t(2 i+3) \cdot v_{i+2}=x(2 k+2 i+2,1) \cdot x(2 k+2 i+1,2) \ldots x(1,2 k+2 i+2)
$$

So if $u=\lambda$ then $\left|g_{i+1}\right|=\left|z_{i+1}\right|+1=2 i+2$ and $t(2 i+3)=x(2 k, 2 i+3)$.

Anf if $u \in \Sigma$ then $\left|g_{i+1}\right|=\left|z_{i+1}\right|+1=2 i+3$ and $t(2 i+3)=x(2 k-1,2 i+4)$.

We have then proved by induction that if $u=\lambda$,

$t=t(1) . t(2) \ldots t(n) \ldots=x(2 k, 1) \cdot x(2 k, 2) \ldots x(2 k, n) \ldots$

and if $u \in \Sigma$,

$t=t(1) . t(2) \ldots t(n) \ldots=x(2 k-1,2) . x(2 k-1,3) \ldots x(2 k-1, n) \ldots$

Notice that in this second case the $\omega$-word $t$ begins with the second letter $x(2 k-$ $1,2)$ of the $(2 k-1)^{t h}$ column of $x$ and not with the first letter $x(2 k-1,1)$ of this column. But this will not change the fact that $t \in \mathcal{A}$ or $t \notin \mathcal{A}$ because $\mathcal{A}$ is simply the set of $\omega$-words over the alphabet $\{0,1\}$ with infinitely many occurrences of the letter 1 .

Thus if a code $h(x)$ of an $\omega^{2}$-word $x \in \Sigma^{\omega^{2}}$ is in $R$ then $x$ has a column in $\mathcal{A}$, i.e. $x \in S$. Conversely it is easy to see that every code $h(x)$ of $x \in S$ may be written in the above form $\left(y_{1}, y_{2}\right)$ of a word in $R$. Then we have proved that $S=h^{-1}(R)$.

Remark that the non determinism of a transducer recognizing $R$ (or of a 2-tape finite automaton accepting $R$ ) will be used to guess the integer $k$ and whether $u=\lambda$ or $u \in \Sigma$.

Intuitively, if $\mathcal{T}$ is such a transducer recognizing $R$ then, during a successful computation accepting the code $h(x)$ of an $\omega^{2}$-word $x$ in $\Sigma^{\omega^{2}}$, the non determinism of $\mathcal{T}$ is used to guess a column of the $\omega^{2}$-word $x$ in order to simulate on this 
column the behaviour of a finite Büchi automaton accepting the $\mathbf{\Pi}_{\mathbf{2}}^{\mathbf{0}}$-complete $\omega$-regular language $\mathcal{A}$.

Lemma 9. The above defined relation $R$ is an infinitary rational relation.

Proof. This is easy to see from the definitions of $R$ and of an infinitary rational relation. The infinitary rational relation $R$ is recognized by the following Büchi transducer $\mathcal{T}=\left(K,(\{0,1, A\}),(\{0,1, A\}), \Delta, q_{0}, F\right)$, where

$$
K=\left\{q_{0}, q_{1}, q_{2}, q_{3}, q_{1}^{0}, q_{1}^{1}, q_{2}^{0}, q_{2}^{1}\right\}
$$

is a finite set of states, $\{0,1, A\}=\Sigma \cup\{A\}$ is the input and the output alphabet (with $\Sigma=\{0,1\}), q_{0}$ is the initial state, and $F=\left\{q_{1}^{1}, q_{2}^{1}\right\}$ is the set of accepting states. Moreover $\Delta \subseteq K \times(\Sigma \cup\{A\})^{\star} \times(\Sigma \cup\{A\})^{\star} \times K$ is the finite set of transitions, containing the following transitions:

$\left(q_{0}, \lambda, a, q_{0}\right)$ and $\left(q_{0}, a, \lambda, q_{0}\right)$, for all $a \in \Sigma$,

$\left(q_{0}, A, A, q_{0}\right)$,

$\left(q_{0}, A, A, q_{1}\right)$,

$\left(q_{1}, u, \lambda, q_{2}\right)$, for all $u \in \Sigma \cup \Sigma^{2}$,

$\left(q_{2}, a, b, q_{2}\right)$, for all $a, b \in \Sigma$,

$\left(q_{2}, A, 0, q_{1}^{0}\right)$ and $\left(q_{2}, A, 1, q_{1}^{1}\right)$,

$\left(q, a, \lambda, q_{3}\right)$, for all $a \in \Sigma$ and $q \in\left\{q_{1}^{0}, q_{1}^{1}\right\}$,

$\left(q_{3}, a, b, q_{3}\right)$, for all $a, b \in \Sigma$,

$\left(q_{3}, 0, A, q_{2}^{0}\right)$ and $\left(q_{3}, 1, A, q_{2}^{1}\right)$,

$\left(q, \lambda, \lambda, q_{2}\right)$, for all $q \in\left\{q_{2}^{0}, q_{2}^{1}\right\}$.

Return now to the proof of Theorem $\left[\right.$ and consider the set $\mathcal{R}=R \cup\left(h\left(\Sigma^{\omega^{2}}\right)\right)^{-}$. It turns out that

$$
\mathcal{R}=\mathcal{S}=h(S) \cup\left(h\left(\Sigma^{\omega^{2}}\right)\right)^{-}
$$

because $S=h^{-1}(R)$. But we have proved that $\left(h\left(\Sigma^{\omega^{2}}\right)\right)^{-}$and $R$ are infinitary rational relations thus $\mathcal{R}=R \cup\left(h\left(\Sigma^{\omega^{2}}\right)\right)^{-}$is the union of two infinitary rational relations hence $\mathcal{R} \in R A T$. Lemma $\left[\right.$ asserts that $\mathcal{R}=\mathcal{S}$ is a $\Sigma_{3}^{0}$-complete subset of $((\Sigma \cup\{A\}) \times(\Sigma \cup\{A\}))^{\omega}$ and this ends the proof.

Remark 10. With a slight modification we could have replaced the set $S$ by the set of $\omega^{2}$-words over $\Sigma$ having at least one column in a given $\boldsymbol{\Pi}_{\mathbf{2}}^{\mathbf{0}}$-complete $\omega$-regular language.

\section{$5 \quad \Pi_{3}^{0}$-complete infinitary rational relations}

We can now state our next result:

Theorem 11. There exist some $\mathbf{\Pi}_{\mathbf{3}}^{\mathbf{0}}$-complete infinitary rational relations. 
Proof. We are going to sketch the proof but we cannot give here all details because of limited space for this paper.

As in the last section, we shall use a well known example of $\Pi_{3}^{0}$-complete set which is a subset of the topological space $\Sigma^{\omega^{2}}$ with $\Sigma=\{0,1\}$.

Recall that the set

$$
P=\left\{x \in\{0,1\}^{\omega^{2}} / \forall m \exists^{<\infty} n x(m, n)=1\right\}
$$

where $\exists<\infty$ means "there exist only finitely many", is a $\Pi_{3}^{0}$-complete subset of $\{0,1\}^{\omega^{2}}$, Kec95, p. 179]. $P=\{0,1\}^{\omega^{2}}-S$ so $P$ is $\Pi_{3}^{0}$-complete" follows directly from "S is $\Sigma_{3}^{0}$-complete".

$P$ is the set of $\omega^{2}$-words having all their columns in the $\boldsymbol{\Sigma}_{\mathbf{2}}^{0}$-complete subset $\mathcal{A}^{-}$ of $\{0,1\}^{\omega}$ where $\mathcal{A}$ is the $\Pi_{2}^{0}$-complete $\omega$-regular language given in Example 4 .

We shall use the same coding $h: x \rightarrow h(x)$ for $\omega^{2}$-words over the alphabet $\Sigma=\{0,1\}$ as in preceding section.

\section{Lemma 12.}

$$
\mathcal{P}=h(P) \cup\left(h\left(\Sigma^{\omega^{2}}\right)\right)^{-}
$$

is a $\mathbf{\Pi}_{\mathbf{3}}^{\mathbf{0}}$-complete subset of $((\Sigma \cup\{A\}) \times(\Sigma \cup\{A\}))^{\omega}$.

Proof. It is similar to proof of Lemma 6 .

We are going to find an infinitary rational relation $R_{1}$ such that $P=h^{-1}\left(R_{1}\right)$. We define now the relation $R_{1}$. It is an $\omega$-language over the alphabet $((\Sigma \cup$ $\{A\}) \times(\Sigma \cup\{A\}))$. Every word of $R_{1}$ may be seen as a pair $y=\left(y_{1}, y_{2}\right)$ of $\omega$-words over the alphabet $\Sigma \cup\{A\}$ and then $y$ is in $R_{1}$ if and only if it is in the form

$y_{1}=U_{k} \cdot u \cdot t(1) \cdot v_{1} \cdot A \cdot g_{1} \cdot t(3) \cdot v_{2} \cdot A \cdot g_{2} \cdot t(5) \ldots A \cdot g_{n} \cdot t(2 n+1) \cdot v_{n+1} \cdot A \ldots$

$y_{2}=V_{k} \cdot u_{1} \cdot t(2) \cdot z_{1} \cdot A \cdot u_{2} \cdot t(4) \cdot z_{2} \cdot A \ldots A \cdot u_{n} \cdot t(2 n) \cdot z_{n} \cdot A \ldots$

where $k$ is an integer $\geq 1, U_{k}, V_{k} \in\left(\Sigma^{\star} . A\right)^{k}, u \in \Sigma^{\star}$, and for all integers $i \geq 1$, $t(i) \in \Sigma$ and

$$
\begin{gathered}
u_{i}, v_{i} \in 0^{\star} \text { and } g_{i}, z_{i} \in \Sigma^{\star} \text { and } \\
\left|v_{i}\right|=\left|u_{i}\right| \quad \text { and } \quad\left[\left|g_{i}\right|=\left|z_{i}\right|+1 \text { or }\left|g_{i}\right|=\left|z_{i}\right|\right]
\end{gathered}
$$

and there exist infinitely many integers $i$ such that $\left|g_{i}\right|=\left|z_{i}\right|$.

Lemma 13. The above defined relation $R_{1}$ satisfies $P=h^{-1}\left(R_{1}\right)$, i.e.:

$$
\forall x \in \Sigma^{\omega^{2}} \quad h(x) \in R_{1} \longleftrightarrow x \in P
$$

Lemma 14. The above defined relation $R_{1}$ is an infinitary rational relation. 
Return to the proof of theorem [1] By Lemma 13 the infinitary relation $R_{1}$ satisfies $P=h^{-1}\left(R_{1}\right)$ thus we shall have

$$
\mathcal{P}=h(P) \cup\left(h\left(\Sigma^{\omega^{2}}\right)\right)^{-}=R_{1} \cup\left(h\left(\Sigma^{\omega^{2}}\right)\right)^{-}
$$

But by Lemma $14 R_{1}$ is rational hence $\mathcal{P}$ is the union of two infinitary rational relations thus $\mathcal{P} \in R A T$ and is $\Pi_{\mathbf{3}}^{\mathbf{0}}$-complete by Lemma 12 .

From Theorems 5 and 11 we can now easily infer the following result:

Corollary 15. There exists some $\boldsymbol{\Delta}_{4}^{0}$ (i.e. $\boldsymbol{\Sigma}_{\mathbf{4}}^{\mathbf{0}} \cap \boldsymbol{\Pi}_{\mathbf{4}}^{0}$ ) infinitary rational relations which are not in $\left(\boldsymbol{\Sigma}_{\mathbf{3}}^{\mathbf{0}} \cup \boldsymbol{\Pi}_{\mathbf{3}}^{\mathbf{0}}\right)$.

The question naturally arises whether there exist some infinitary rational relations $R \subseteq \Sigma^{\omega} \times \Gamma^{\omega}$ which are $\boldsymbol{\Sigma}_{4}^{0}$-complete or $\boldsymbol{\Pi}_{4}^{\mathbf{0}}$-complete or even higher in the Borel hierarchy.

Acknowledgements. Thanks to Jean-Pierre Ressayre and Pierre Simonnet for useful discussions and to the anonymous referees for useful comments on a previous version of this paper.

\section{References}

BT70. Ya M. Barzdin and B.A. Trakhtenbrot, Finite Automata, Behaviour and Synthesis, Nauka, Moscow, 1970 (English translation, North Holland, Amsterdam, 1973).

BCPS00. M.-P. Béal, O. Carton, C. Prieur and J. Sakarovitch, Squaring Transducers: An Efficient Procedure for Deciding Functionality and Sequentiality, Theoretical Computer Science, vol. 292, no. 1, pp. 45-63, 2003.

Ber79. J. Berstel, Transductions and Context Free Languages, Teubner Verlag, 1979.

Büc62. J.R. Büchi, On a Decision Method in Restricted Second Order Arithmetic, Logic Methodology and Philosophy of Science, ( Proc. 1960 Int. Congr. ), Stanford University Press, 1962, 1-11.

CDT02. T. Cachat, J. Duparc and W. Thomas, Solving Pushdown Games with a $\Sigma_{3}$ Winning Condition, proceedings of CSL 2002, LNCS 2471, pp. 322-336,

Cho77. C. Choffrut, Une Caractérisation des Fonctions Séquentielles et des Fonctions Sous-Séquentielles en tant que Relations Rationnelles, Theoretical Computer Science, Volume 5, 1977, p.325-338.

CG99. C. Choffrut and S. Grigorieff, Uniformization of Rational Relations, Jewels are Forever 1999, J. Karhumki, H. Maurer, G. Paun and G. Rozenberg editors, Springer, p.59-71.

EH93. J. Engelfriet and H. J. Hoogeboom, X-automata on $\omega$-Words, Theoretical Computer Science 110 (1993) 1, 1-51.

Fin01a. O. Finkel, Topological Properties of Omega Context Free Languages, Theoretical Computer Science, Vol 262 (1-2), July 2001, p. 669-697.

Fin01b. O. Finkel, On the Topological Complexity of Infinitary Rational Relations, RAIRO-Theoretical Informatics and Applications, to appear.

FS93. C. Frougny and J. Sakarovitch, Synchronized Rational Relations of Finite and Infinite Words, Theoretical Computer Science 108 (1993) 1, p.45-82. 
Gir81. F. Gire, Relations Rationnelles Infinitaires, Thèse de troisième cycle, Université Paris 7, Septembre 1981.

Gir83. F. Gire, Une Extension aux Mots Infinis de la Notion de Transduction Rationnelle, 6th GI Conf., Lect. Notes in Comp. Sci., Volume 145, 1983, p. 123-139.

GN84. F. Gire and M. Nivat, Relations Rationnelles Infinitaires, Calcolo, Volume XXI, 1984, p. 91-125.

Kec95. A.S. Kechris, Classical Descriptive Set Theory, Springer-Verlag, 1995.

Lan69. L. H. Landweber, Decision Problems for $\omega$-Automata, Math. Syst. Theory 3 (1969) 4,376-384.

LT94. H. Lescow and W. Thomas, Logical Specifications of Infinite Computations, In:" A Decade of Concurrency" (J. W. de Bakker et al., eds), Springer LNCS 803 (1994), 583-621.

LS77. R. Lindner and L. Staiger, Algebraische Codierungstheorie - Theorie der Sequentiellen Codierungen, Akademie-Verlag, Berlin, 1977.

Mos80. Y. N. Moschovakis, Descriptive Set Theory, North-Holland, Amsterdam 1980.

PP01. D. Perrin and J.-E. Pin, Infinite Words, Book in preparation, available from http://www.liafa.jussieu.fr/jep/InfiniteWords.html

Pin96. J-E. Pin, Logic, Semigroups and Automata on Words, Annals of Mathematics and Artificial Intelligence 16 (1996), p. 343-384.

Pri00. C. Prieur, Fonctions Rationnelles de Mots Infinis et Continuité, Thèse de Doctorat, Université Paris 7, Octobre 2000.

Sim92. P. Simonnet, Automates et Théorie Descriptive, Ph.D. Thesis, Université Paris 7, March 1992.

Sta86. L. Staiger, Hierarchies of Recursive $\omega$-Languages, Jour. Inform. Process. Cybernetics EIK 22 (1986) 5/6, 219-241.

Sta97. L. Staiger, $\omega$-Languages, Chapter of the Handbook of Formal languages, Vol 3, edited by G. Rozenberg and A. Salomaa, Springer-Verlag, Berlin.

SW78. L. Staiger and K. Wagner, Rekursive Folgenmengen I, Z. Math Logik Grundlag. Math. 24, 1978, 523-538.

Tho89. W. Thomas, Automata and Quantifier Hierarchies, in: Formal Properties of Finite automata and Applications, Ramatuelle, 1988, Lecture Notes in Computer Science 386, Springer, Berlin, 1989, p.104-119.

Tho90. W. Thomas, Automata on Infinite Objects, in: J. Van Leeuwen, ed., Handbook of Theoretical Computer Science, Vol. B (Elsevier, Amsterdam, 1990 ), p. 133-191. 